\title{
The prognostic value of CXC-chemokine receptor 2 (CXCR2) in gastric cancer patients

\author{
Zhenglin Wang ${ }^{1,2 \dagger}$, Hao Liu ${ }^{1 \dagger}$, Zhenbin Shen ${ }^{1 \dagger}$, Xuefei Wang ${ }^{1}$, Heng Zhang ${ }^{1}$, Jing Qin ${ }^{1}$, Jiejie Xu ${ }^{3 *}$, \\ Yihong Sun ${ }^{1 *}$ and Xinyu Qin ${ }^{1 *}$
}

\begin{abstract}
Background: CXC chemokine receptor 2 (CXCR2) has been reported to play an important role in the proliferation and invasion of gastric cancer cells. The present study aims to investigate the impact of CXCR2 expression on the overall survival (OS) of gastric cancer patients after radical resection.

Methods: Intratumoral CXCR2 expression was evaluated with immunohistochemistry on tissue microarrays containing tumor samples of 357 gastric cancer patients from a single center. CXCR2 expression levels were correlated to clinicopathological variables and OS.
\end{abstract}

Results: CXCR2 expression was mainly located in the cytoplasm of gastric carcinoma cells. High CXCR2 expression was associated with poor tumor differentiation $(p=0.021)$, increased tumor depth $(p<0.001)$, lymph node metastasis $(p<0.001)$, advanced TNM stage $(p<0.001)$ and short OS $(p=0.001)$. CXCR2 expression was an independent prognostic factor for OS $(p=0.001)$ in multivariate analysis, and could be combined with TNM stage to generate a predictive nomogram for clinical outcome in patients with gastric cancer.

Conclusion: Intratumoral CXCR2 expression is a novel independent predictor for survival in gastric cancer patients. CXCR2 might be a promising therapeutic target of postoperative adjuvant treatment.

Keywords: Gastric cancer, CXC chemokine receptor 2, Prognosis, Nomogram, Overall survival

\section{Background}

Despite the incidence of gastric cancer has declined in the modern society for decades, it remains the fourth most common malignancy and the third leading cause of cancer related death worldwide with an estimated 951,600 new cases and 723,100 deaths occurring in 2012 [1]. A substantial proportion of gastric cancer patients are diagnosed at advanced stages, due to occult symptoms at early stages, whereas patients in Japan gain a 5 -year overall survival as high as $76 \%$, attributing to the screening for early stage gastric cancer [2]. Currently, prognostic models for gastric cancer are mainly based on the TNM classification of International

\footnotetext{
* Correspondence: jjxufdu@fudan.edu.cn; sun.yihong@zs-hospital.sh.cn; qin. xinyu@zs-hospital.sh.cn

†Equal contributors

${ }^{3}$ Department of Biochemistry and Molecular Biology, School of Basic Medical Sciences, Fudan University, PO Box 103138 Yi Xue Yuan Road, Shanghai 200032, China

'Department of General Surgery, Zhongshan Hospital, Fudan University, 180 Feng Lin Road, Shanghai 200032, China

Full list of author information is available at the end of the article
}

Union Against Cancer, composed of tumor depth, lymph node metastasis and distant metastasis. The outcomes for patients with similar pathological TNM stage can be very diverse because of the heterogeneity of this disease [3, 4]. Therefore, stratifying patients in the current TNM stage system by incorporation of the molecules involved in carcinogenesis of gastric cancer may lead to more accurate prediction of the clinical outcome.

Chemokines are a superfamily of small molecule proteins and selectively regulate the recruitment and activation of leukocyte subsets to preferential sites through chemotaxis [5]. CXCR2 is a member of the Gprotein-coupled receptor superfamily and the receptor for chemokines with the presence or absence of ELR motif (Glu-Leu-Arg). The ELR positive CXC chemokines (such as CXCL1, CXCL2, CXCL3, CXCL5, CXCL6, CXCL8 and CXCL7) are potent promoters of angiogenesis $[6,7]$. A number of studies have demonstrated that CXCR2 plays a pivotal role in tumor 
angiogenesis, proliferation and invasion [8-10]. In gastric cancer, CXCR2 was found to be associated with tumor progression and invasion [11, 12]. Thus, we hypothesized that the addition of CXCR2 to TNM staging system has the potential to provide more individualized risk stratification based on molecular characteristics of the tumor.

In this study, we investigated CXCR2 expression in patients with gastric cancer by immunohistochemistry and explored its associations with clinicopathological factors and prognosis. Moreover, we generated a predictive nomogram integrating CXCR2 expression, tumor depth, and lymph node metastasis to assess the risk score for 5-year overall survival (OS) of gastric cancer patients.

\section{Methods}

\section{Patients}

We retrospectively recruited 357 consecutive gastric cancer patients from Zhongshan Hospital, Fudan University. Gastrectomy plus standard D2 lymphadenectomy was performed by the same surgical team in 2008. None of these patients received any preoperative chemotherapy or radiotherapy. Baseline clinicopathological features of these patients including age, gender, tumor location, tumor size, tumor differentiation, Lauren classification, and TNM stage were collected. Tumor stage and differentiation grade were reassessed according to the 7th Edition of the UICC/AJCC TNM Staging System by two independent gastroenterological pathologists. Median age at surgery was 59 years (range $27-85$ ), and $70 \%$ of patients were male. Intestinal and diffuse histologic subtypes constituted $63 \%$ and $37 \%$ of cases, respectively. Lymph node metastasis was present in $64 \%$ of patients. Patients were followed up until April 2014 with a median follow-up time of 41 months. Overall survival was defined as the interval between the date of surgery and the date of death or last visit. The study was approved by the Clinical Research Ethics Committee of Zhongshan Hospital, Fudan University and written informed consent was obtained from each patient.

\section{Tissue microarray, immunohistochemical staining and evaluation}

Tissue microarray construction and immunohistochemistry protocol were described previously [13]. The primary antibody against human CXCR2 (Abcam, Cambridge, MA, USA; dilution 1:100) was applied in the procedure. The staining intensity and extent were scored independently by two gastroenterological pathologists ( $Z$. Shen and $H$. Zhang) who were blind to the patients' outcome using the semi-quantitative immunoreactivity scoring (IRS) system as described previously [14]. The immunohistochemicalstained sections were scanned at $\times 200$ magnification and three independent microscopic fields with the strongest staining were captured by NIS-Element F3.2 software to guarantee representativeness and homogeneity. The staining intensity was graded as 0 (negative), 1 (weak), 2 (moderate), and 3 (strong) and the staining extent was scored as the percentage of positive cells $(0-100 \%)$. The staining intensity and extent were multiplied to obtain a CXCR2 immunohistochemical score on a scale of 0 to 300. The agreement among the two pathologists was excellent, which was evaluated by the kappa value (0.85). To dichotomize CXCR2 expression into high and low groups, the score of 200 was selected as the cutoff point according to the minimum $p$-value method based on its correlation with OS. The negative control staining was treated equally with the primary antibody excluded.

\section{Statistical analysis}

SPSS 21.0 (SPSS Inc., IL, Chicago, USA) was used to perform the analyses. Correlations between immunohistochemical variables and clinicopathologic characteristics were analyzed with Pearson $\chi^{2}$ and Student's $t$ tests. Kaplan-Meier method with log-rank test was applied to compare survival curves. Cox regression models were used to analyze the impact of prognostic factors on OS. Nomogram was constructed by $\mathrm{R}$ software version 3.0.2 with "rms" package ( $\mathrm{R}$ Foundation for Statistical Computing, Vienna, Austria). Calibration plot for 5-year overall survival was generated to assess the performance characteristics of the constructed nomogram. The Harrell's concordance indices (c-indices) were calculated to evaluate the discrimination of different models for OS prediction. All statistical analyses were two-sided and $p<0.05$ was regarded as statistically significant.

\section{Results}

\section{CXCR2 expression and associations with}

clinicopathological features in gastric cancer patients

Immunohistochemical staining section analysis demonstrated that CXCR2 expression was mainly located in the cytoplasm of gastric carcinoma cells (Fig. 1b-d). The median intratumoral CXCR2 staining score was 210 (range 0-300). The negative control showed no staining neither in gastric epithelial cells nor in stroma cells (Fig. 1a). The relationships between clinical pathological characteristics and CXCR2 expression are shown in Table 1. High CXCR2 expression correlated with poor tumor differentiation $(p=0.021)$, increased tumor depth $(p<0.001)$, lymph node metastasis $(p<0.001)$ and advanced TNM stage $(p<0.001)$.

\section{High expression of CXCR2 is associated with poor clinical outcome}

The Kaplan-Meier curves revealed that high CXCR2 expression correlated with shorter OS $(p<0.001$, Fig. 2a). The median survival time for CXCR2 high and low expression group was 32 and 51 months, respectively. 

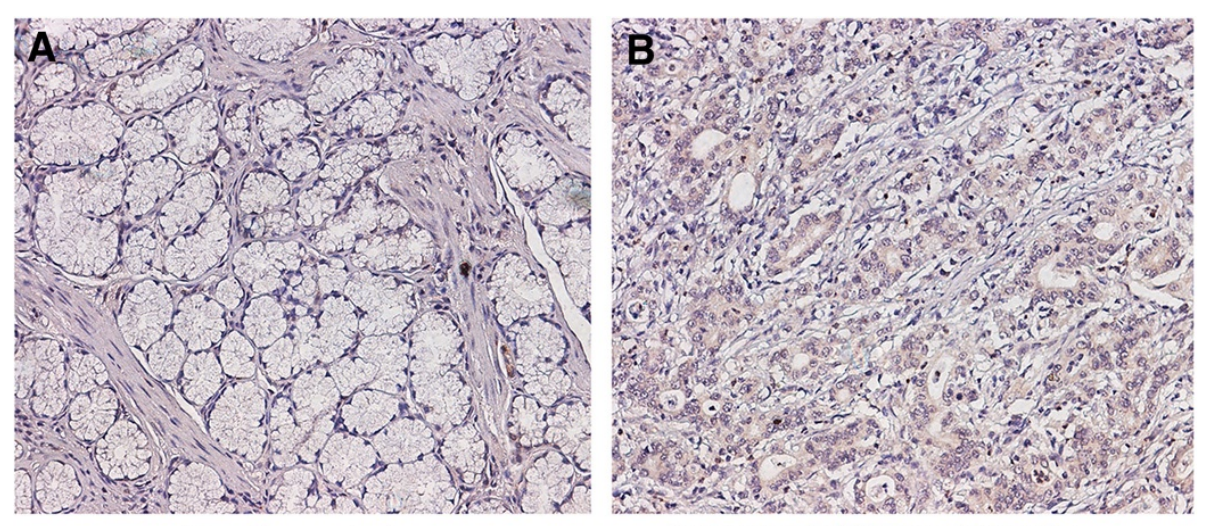

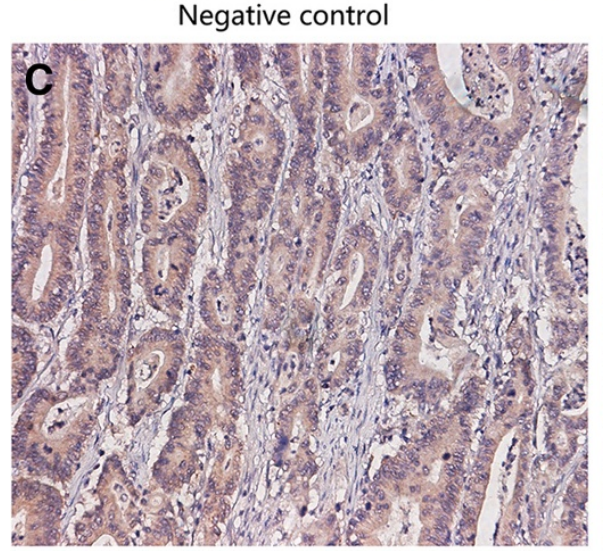

Intratumoral CXCR2 moderate intensity
Intratumoral CXCR2 weak intensity

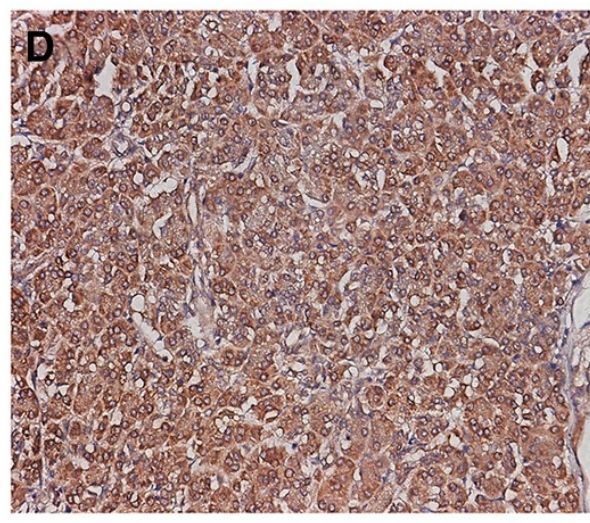

Intratumoral CXCR2 strong intensity

Fig. 1 Expression of CXCR2 in sections of gastric cancer. Representative photographs of CXCR2 expression (a-d). Negative control a. Representative photographs of weak, moderate and strong staining (b-d). Original magnification: $\times 200$

Subgroup analysis revealed that intratumoral CXCR2 expression played an unfavorable prognostic role in patients of T3 ( $p=0.001$, Fig. 2e), T4 ( $p=0.005$, Fig. 2f), N0 ( $p=0.003$, Fig. 3a), moderate differentiation $(p=0.019$, Fig. 2c), poor differentiation ( $p<0.001$, Fig. $2 d)$, TNM I + II $(p=0.002$, Fig. 3c), TNM III + IV ( $p=0.008$, Fig. 3d), Lauren intestinal type $(p<0.01$, Additional file 1: Figure S1E) and Lauren diffuse type ( $p=0.012$, Additional file 1: Figure S1F). In contrast, intratumoral CXCR2 expression had limited ability to stratify patients with T1, T2, N1, N2, N3 and well differentiated disease. (Figure 2b, Additional file 1: Figure S1A-D). To further elucidate the predictive value of CXCR2 precisely, we calculated its hazard ratios (HR) using univariate COX regression in different subgroups and found that CXCR2 expression exerted the same adverse prognostic role as it did in Log-rank test (Additional file 2: Figure S2).

\section{Multivariate analysis and predictive nomogram for OS of gastric cancer patients}

We then evaluated the independent prognostic value of CXCR2 expression using multivariate Cox proportional hazard model. The results showed that the CXCR2 expression was independently prognostic of mortality $(\mathrm{HR}=1.860 ; 95 \% \mathrm{CI}=1.343-2.575 ; p<0.001)$ in patients with gastric cancer after adjusting for established clinicopathologic factors (Fig. 4a).

Predictive nomogram was constructed using all the significant independent predictors for OS from Cox regression analysis. In the nomogram, the hazard ratio for each factor was turned into points, and a higher total points indicated worse survival overall probability (Fig. 4b). The calibration curve for predicted 5-year OS showed a good performance with the ideal model (Fig. 4c). The Harrell's concordance index (c-index) for the nomogram constructed by TNM and CXCR2 expression was 0.664 , higher than 0.642 of TNM alone.

\section{Discussion}

CXCR2 expression has been implicated in gastric cancer progression [11, 12, 15, 16]. Coexpression of CXCL1 and CXCR2 acts like an autocrine or paracrine mechanism to actuate metastasis of gastric cancer [15]. However, its prognostic value in gastric cancer patients has not been well established. In this study, we investigated the expression of this chemokine receptor with 
Table 1 Relation between intratumoral CXCR2 expression and clinical characteristics in patients with gastric cancer $(n=357)$

\begin{tabular}{|c|c|c|c|c|c|}
\hline \multirow[t]{2}{*}{ Factor } & \multicolumn{2}{|l|}{ Patients } & \multicolumn{3}{|c|}{ CXCR2 expression } \\
\hline & No. & $\%$ & Low & High & $P$ value \\
\hline All patients & 357 & 100 & 157 & 200 & \\
\hline Age (years) & & & & & 0.250 \\
\hline Mean $\pm \mathrm{SD}^{\dagger}$ & $59.4 \pm 11.6$ & & $58.6 \pm 11.1$ & $60.1 \pm 11.9$ & \\
\hline Gender & & & & & 0.477 \\
\hline Female & 107 & 30 & 44 & 63 & \\
\hline Male & 250 & 70 & 113 & 137 & \\
\hline Tumor size (cm) & & & & & 0.931 \\
\hline Mean \pm SD + & $3.8 \pm 2.1$ & & $3.8 \pm 2.4$ & $3.8 \pm 1.9$ & \\
\hline Differentiation & & & & & 0.021 \\
\hline Well & 20 & 5.6 & 14 & 6 & \\
\hline Moderately & 127 & 35.6 & 60 & 67 & \\
\hline Poorly & 210 & 58.8 & 83 & 127 & \\
\hline Lauren classification & & & & & 0.910 \\
\hline Intestinal & 224 & 62.7 & 98 & 126 & \\
\hline Diffuse & 133 & 37.3 & 59 & 74 & \\
\hline pT stage & & & & & $<0.001$ \\
\hline $\mathrm{T} 1$ & 60 & 16.8 & 40 & 20 & \\
\hline $\mathrm{T} 2$ & 50 & 14.0 & 25 & 25 & \\
\hline $\mathrm{T} 3$ & 65 & 18.2 & 27 & 38 & \\
\hline T4 & 182 & 51.0 & 65 & 117 & \\
\hline pN stage & & & & & $<0.001$ \\
\hline No & 128 & 35.8 & 72 & 56 & \\
\hline N1 & 37 & 10.4 & 21 & 16 & \\
\hline $\mathrm{N} 2$ & 70 & 19.6 & 24 & 46 & \\
\hline N3 & 122 & 34.2 & 40 & 82 & \\
\hline Distant metastasis & & & & & 0.735 \\
\hline Absent & 349 & 97.8 & 153 & 196 & \\
\hline Present & 8 & 2.2 & 4 & 4 & \\
\hline TNM stage & & & & & 0.001 \\
\hline 1 & 78 & 21.8 & 49 & 29 & \\
\hline$\|$ & 80 & 22.5 & 37 & 43 & \\
\hline III & 191 & 53.5 & 67 & 124 & \\
\hline IV & 8 & 2.2 & 4 & 4 & \\
\hline
\end{tabular}

${ }^{*} p<0.05$ was regarded as statistically significant. ${ }^{\dagger} \mathrm{SD}$ : standard deviation

immunohistochemistry on gastric cancer tissue microarray and its relationships with pathologic factors and prognosis. Our data showed a consistent result with previous studies that CXCR2 expression positively correlated with tumor depth, lymph node metastasis and TNM stage (Table 1), which implied that CXCR2 expression might synergize gastric cancer proliferation, invasion and metastasis.

Notably, an interesting phenomenon had been observed that CXCR2 staining increased gradually accompanied with gastric cancer differentiation from well to poor (Fig. 1b-d). This raised the possibility that CXCR2 expression increases during the dedifferentiation process of gastric cancer cells and might take on a particular role in gastric cancer differentiation. However, the biological mechanisms underlying this phenomenon merit further investigation.

The connection between inflammation and cancer was first noted by Virchwood in the 19th century [13]. Chronic inflammation is frequently present before several types of carcinogenesis. Chemokines are the key molecule for 


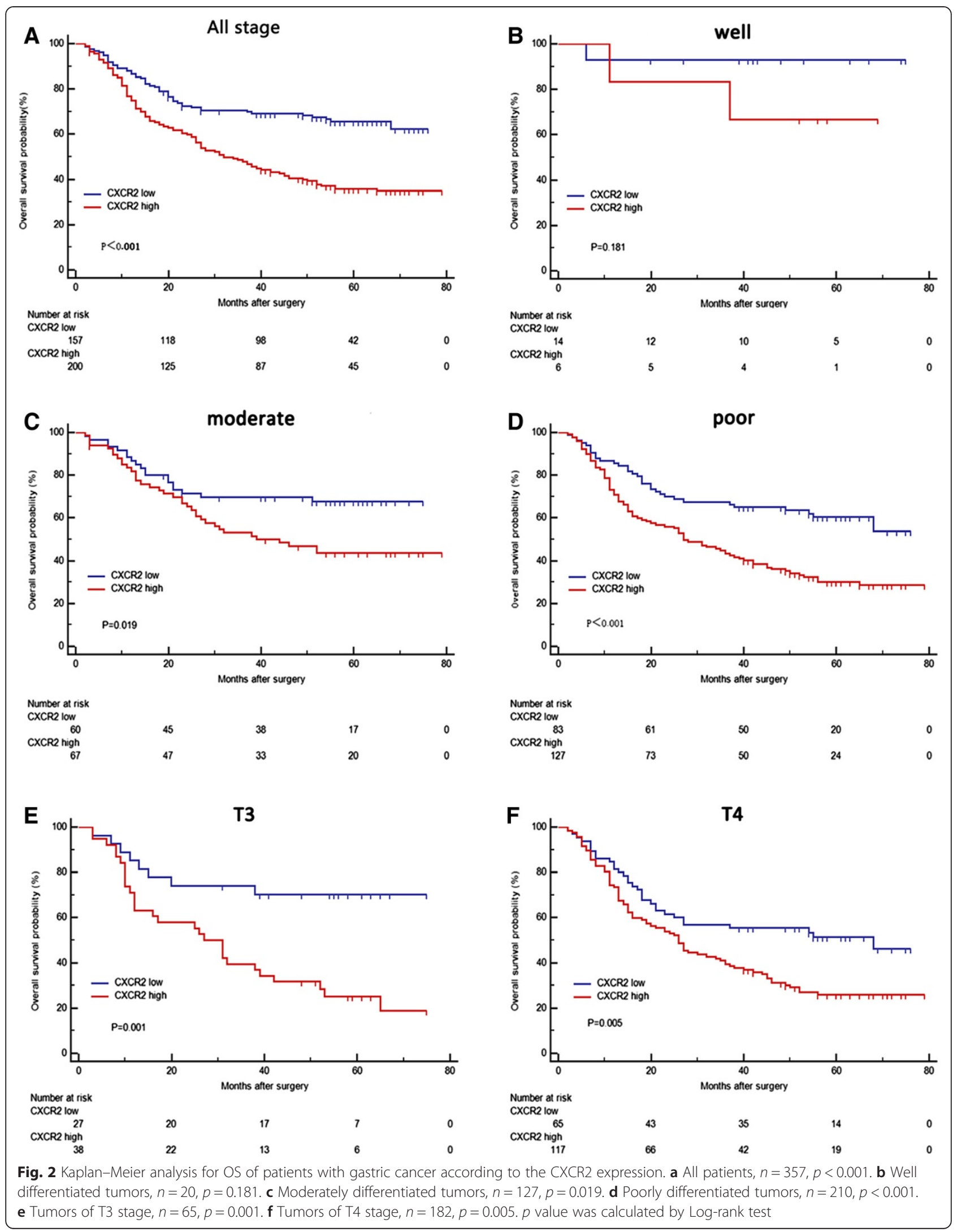




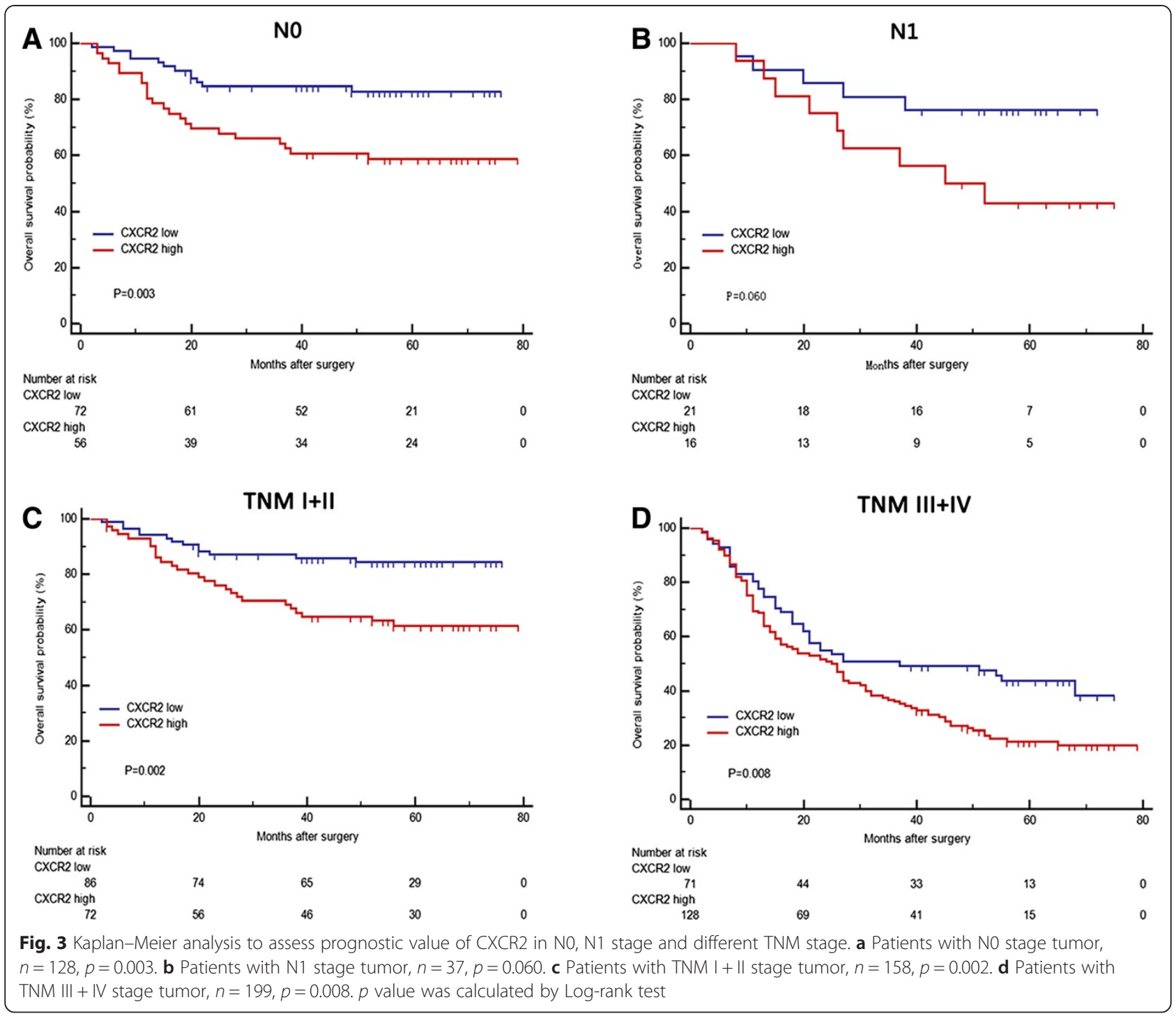

inducing leukocytes to inflammation or tumor site [1719]. Expression of pro-inflammatory chemokines facilitates a chronic inflammation process and helps establish a favorable tumor milieu, which stimulates tumor proliferation and invasion via their receptors on tumor cells [20, 21]. Many studies have identified that ELR positive chemokines play a pleiotropic role in inflammation, angiogenesis, carcinogenesis and metastasis [22-24].

CXCR2 was reported to play a critical role in a range of cancers, such as colon cancer [25], oral squamous cell cancer [26], esophageal cancer [27] and breast cancer [28]. CXCR2 had been found to be the primary functional chemokine receptor in mediating endothelial cell chemotaxis [29]. All ELR+ CXC chemokine ligands, binding to CXCR2, mediated angiogenic activity, which was crucial for cancer cells proliferation [22]. Heidemann found that after activation of CXCR2 using interleukin-8 (IL-8), endothelial cells gained enhanced capacity of fiber assembly, proliferation and phosphorylation of its downstream signaling molecule ERK1/2 while this phenomenon could be impaired by either using specific antibodies to CXCR2 or inhibitor for ERK1/2 [24, 25]. The importance of CXCR2 in angiogenesis in vivo had also been proven in the cornea micropocket assay by CXCR2 knockout mice [22]. Thus, the correlation between aberrant expression of CXCR2 and the poor prognosis of the patients was possibly due to its angiogenic role in gastric cancer.

Although several biomarkers have been introduced to the prognosis models for gastric cancer recently $[30,31]$, conventional predictive models majorly rely on TNM stage, which has limited ability to discriminate a stratum of patients for the heterogeneity of this disease. Kaplan-Meier and univariate COX stratification analysis revealed that CXCR2 had a discriminatory power in most subgroups of different clinicopathological types (Additional file 2: Figure S2). Further analysis of 


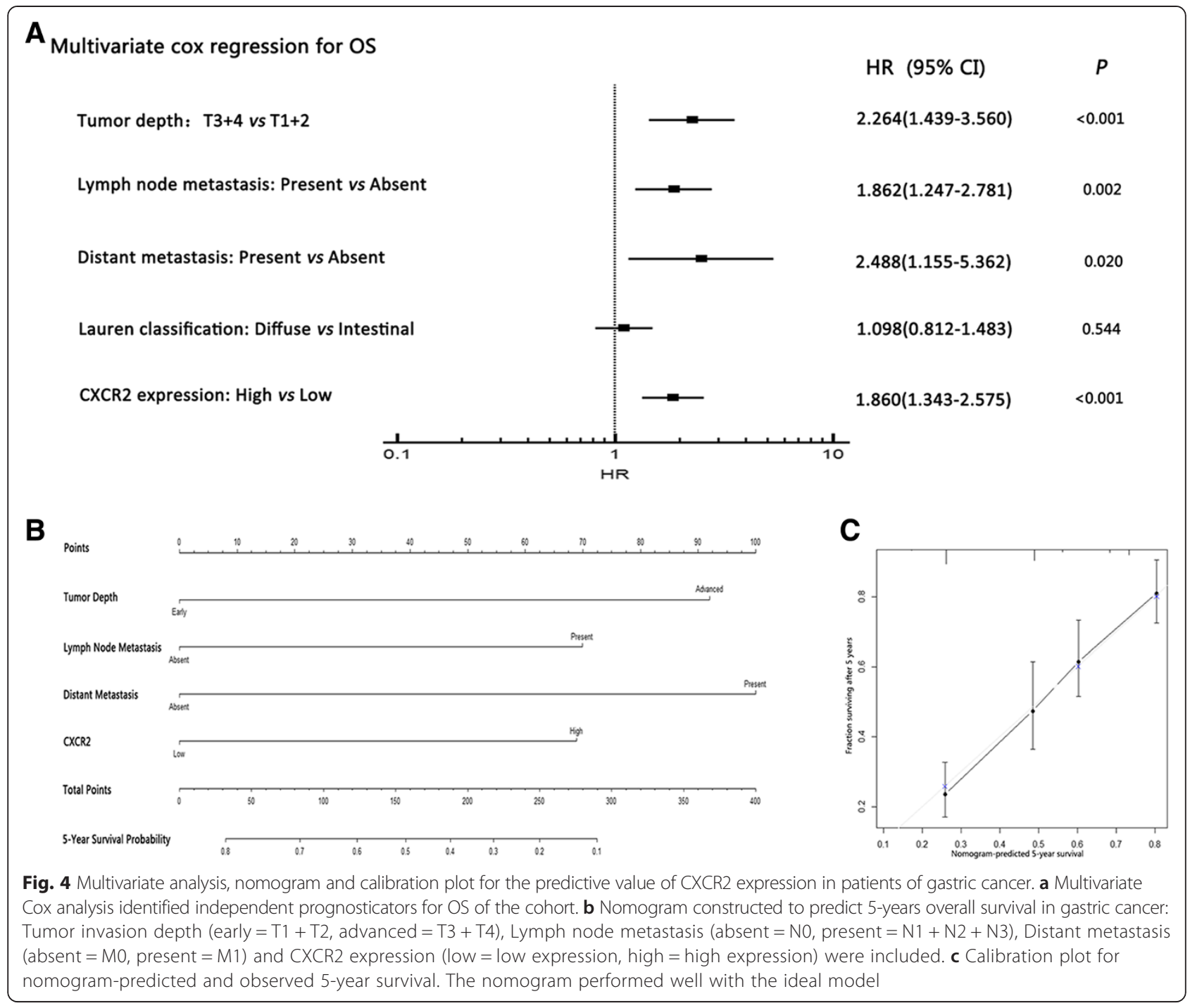

multivariate COX regression verified that CXCR2 bears an independent prognostic value, which could be integrated to the TNM staging system in the nomogram (Fig. 4a-c). Validation test using calibration plot and cindex indicated that this nomogram performed better than the TNM stage alone.

Giving the prognostic value of CXCR2 expression in gastric cancer, optimal use of CXCR2 inhibitors would be a potential choice of adjuvant therapy for gastric cancer patients after gastrectomy. However, due to the retrospective design in nature and the relatively small size of the patient population, a multicenter, prospective study is needed to validate these results in a larger population in the future.

\section{Conclusion}

Our present study identified that intratumoral CXCR2 expression correlates with gastric cancer progression, tumor differentiation and lymph node metastasis and can be utilized as a novel prognostic factor for patient outcomes. Incorporating CXCR2 expression into TNM stage can provide a better prognostic model for patients with gastric cancer. Inhibition of CXCR2 might be a promising target of postoperative adjuvant therapy modality for gastric cancer patients.

\section{Additional files}

Additional file 1: Figure S1. Kaplan-Meier analysis to assess prognostic value of CXCR2 in some clinicopathological factors. (A) T1 stage, $n=80$, $p=0.386$. (B) T2 stage, $n=50, p=0.803$. (C) N2 stage, $n=70, p=0.124$. (D) N3 stage, $n=122, p=0.162$. (E) Lauren intestinal type, $n=224, p<0.01$. (F) Lauren diffuse type, $n=133, p=0.012$. (JPEG $322 \mathrm{~kb}$ )

Additional file 2: Figure S2. COX analysis assesses prognostic value of CXCR2 with hazard ratios for OS in subgroups. T3 ( $n=65$, HR: $3.326,95 \%$ Cl: $1.522-7.267, p=0.003)$, T4 $(n=182, \mathrm{HR}: 1.768,95 \% \mathrm{Cl}: 1.178-2.652$, $p=0.006)$, NO $(n=128$, HR: $2.782,95 \% \mathrm{Cl}: 1.389-5.574, p=0.004)$, TNM I + II $(n=158$, HR: $2.713,95 \%$ Cl: 1.404-5.241, $p=0.003)$, TNM III + IV ( $n=199, \mathrm{HR}: 1.623,95 \% \mathrm{Cl}: 1.126-2.340, p=0.01$ ), well and moderate differentiation ( $n=147, \mathrm{HR}: 2.159,95 \% \mathrm{Cl}$ : 1.262-3.691, $p=0.005)$, poor differentiation ( $n=210$, HR: $2.158,95 \% \mathrm{Cl}: 1.448-3.217, p<0.001$ ), 
Lauren intestinal type ( $n=224, \mathrm{HR}: 2.573,95 \% \mathrm{Cl}: 1.672-3.958, p<0.001)$, Lauren diffuse type ( $n=133$, HR: 1.834, $95 \%$ Cl: 1.137-2.960, $p=0.014)$. (JPEG $302 \mathrm{~kb}$ )

\section{Abbreviations}

CXCR2: C-X-C chemokine receptor 2; CXCL: C-X-C chemokine ligand; OS: Overall survival; UICC: International Union Against Cancer; AJCC: American Joint Committee on Cancer; IL8: Interleukin 8; ERK1/2: Extracellular signal-regulated kinase1/2; c-index: Harrell's concordance index.

\section{Competing interests}

The authors have declared no conflicts of interest.

\section{Authors' contributions}

ZW designed and conducted experiments, performed statistical analysis and drafted the manuscript. HL carried out laboratory work and data analysis. ZS performed the pathological analysis and was involved in the collection of patient materials and drafting of the manuscript. XW participated in the study design and collection of related articles. $\mathrm{HZ}$ performed the pathological analysis and laboratory work. JQ performed laboratory work and was in charge of correction of words in the manuscript. JX was responsible for the acquisition of related articles and revising manuscript critically for important intellectual content. YS conceived the design of this study, lead the data analysis and oversaw the drafting of the manuscript. XQ took charge of study design and revising of the manuscript. All authors read and approved the final manuscript.

\section{Acknowledgements}

This study was funded by grants from National Natural Science Foundation of China (31100629, 31270863, 31300671, 31470794, 81471621, 81472227), Program for New Century Excellent Talents in University (NCET-13-0146) and Shanghai Rising-Star Program (13QA1400300). All these study sponsors have no roles in the study design, in the collection, analysis, and interpretation of data.

\section{Author details}

'Department of General Surgery, Zhongshan Hospital, Fudan University, 180 Feng Lin Road, Shanghai 200032, China. 'Department of Gastroenterological Surgery, The First Affiliated Hospital of Dalian Medical University, Dalian 116011, China. ${ }^{3}$ Department of Biochemistry and Molecular Biology, School of Basic Medical Sciences, Fudan University, PO Box 103138 Yi Xue Yuan Road, Shanghai 200032, China.

Received: 14 May 2015 Accepted: 15 October 2015

Published online: 23 October 2015

\section{References}

1. Torre LA, Bray F, Siegel RL, Ferlay J, Lortet-Tieulent J, Jemal A. Global cancer statistics, 2012. CA Cancer J Clin. 2015;65(2):87-108.

2. Yamada T, Yoshikawa T, Taguri M, Hayashi T, Aoyama T, Sue-Ling HM, Bonam K, Hayden JD, Grabsch HI: The survival difference between gastric cancer patients from the UK and Japan remains after weighted propensity score analysis considering all background factors. Gastric cancer. 2015. Epub ahead of print.

3. Lim L, Michael M, Mann GB, Leong T. Adjuvant therapy in gastric cancer. J Clin Oncol. 2005;23(25):6220-32.

4. Stock M, Otto F. Gene deregulation in gastric cancer. Gene. 2005;360(1):1-19.

5. Richmond A. Chemokine research moves on. Exp Cell Res. 2011;317(5):553-5.

6. Strieter RM, Polverini PJ, Kunkel SL, Arenberg DA, Burdick MD, Kasper J, et al. The functional role of the ELR motif in CXC chemokine-mediated angiogenesis. J Biol Chem. 1995;270(45):27348-57.

7. Strieter RM, Belperio JA, Phillips RJ, Keane MP. CXC chemokines in angiogenesis of cancer. Semin Cancer Biol. 2004;14(3):195-200.

8. Saintigny P, Massarelli E, Lin S, Ahn YH, Chen Y, Goswami S, et al. CXCR2 expression in tumor cells is a poor prognostic factor and promotes invasion and metastasis in lung adenocarcinoma. Cancer Res. 2013;73(2):571-82.

9. Grepin R, Guyot M, Giuliano S, Boncompagni M, Ambrosetti D, Chamorey E, et al. The CXCL7/CXCR1/2 axis is a key driver in the growth of clear cell renal cell carcinoma. Cancer Res. 2014;74(3):873-83.
10. Sui P, Hu P, Zhang T, Zhang X, Liu Q, Du J. High expression of CXCR-2 correlates with lymph node metastasis and predicts unfavorable prognosis in resected esophageal carcinoma. Med Oncol. 2014;31(2):809.

11. Wang JP, Hu WM, Wang KS, Yu J, Luo BH, Wu C, et al. Expression of C-X-C chemokine receptor types $1 / 2$ in patients with gastric carcinoma: Clinicopathological correlations and significance. Oncol Lett. 2013;5(2):574-82.

12. Li Z, Wang Y, Dong S, Ge C, Xiao Y, Li R, et al. Association of CXCR1 and 2 expressions with gastric cancer metastasis in ex vivo and tumor cell invasion in vitro. Cytokine. 2014;69(1):6-13.

13. Mantovani A, Allavena P, Sica A, Balkwill F. Cancer-related inflammation. Nature. 2008;454(7203):436-44.

14. Weichert W, Roske A, Gekeler V, Beckers T, Ebert MP, Pross M, et al. Association of patterns of class I histone deacetylase expression with patient prognosis in gastric cancer: a retrospective analysis. Lancet Oncol. 2008;9(2):139-48.

15. Cheng WL, Wang CS, Huang YH, Tsai MM, Liang Y, Lin KH. Overexpression of CXCL1 and its receptor CXCR2 promote tumor invasion in gastric cancer. Ann Oncol. 2011;22(10):2267-76.

16. Wei ZW, Xia GK, Wu Y, Chen W, Xiang Z, Schwarz RE, et al. CXCL1 promotes tumor growth through VEGF pathway activation and is associated with inferior survival in gastric cancer. Cancer Lett. 2015;359(2):335-43.

17. Mantovani A. The chemokine system: redundancy for robust outputs. Immunol Today. 1999;20(6):254-7.

18. Allavena P, Germano G, Marchesi F, Mantovani A. Chemokines in cancer related inflammation. Exp Cell Res. 2011;317(5):664-73.

19. Lazennec $G$, Richmond A. Chemokines and chemokine receptors: new insights into cancer-related inflammation. Trends Mol Med. 2010;16(3):133-44.

20. Vandercappellen J, Van Damme J, Struyf S. The role of CXC chemokines and their receptors in cancer. Cancer Lett. 2008;267(2):226-44.

21. Verbeke H, Geboes K, Van Damme J, Struyf S. The role of CXC chemokines in the transition of chronic inflammation to esophageal and gastric cancer. Biochim Biophys Acta. 2012;1825(1):117-29.

22. Addison CL, Daniel TO, Burdick MD, Liu H, Ehlert JE, Xue YY, et al. The CXC chemokine receptor 2, CXCR2, is the putative receptor for ELR+ CXC chemokine-induced angiogenic activity. J Immunol. 2000;165(9):5269-77.

23. Varney ML, Singh S, Li A, Mayer-Ezell R, Bond R, Singh RK. Small molecule antagonists for CXCR2 and CXCR1 inhibit human colon cancer liver metastases. Cancer Lett. 2011;300(2):180-8.

24. Singh S, Nannuru KC, Sadanandam A, Varney ML, Singh RK. CXCR1 and CXCR2 enhances human melanoma tumourigenesis, growth and invasion. Br J Cancer. 2009;100(10):1638-46.

25. Heidemann J, Ogawa H, Dwinell MB, Rafiee P, Maaser C, Gockel HR, et al. Angiogenic effects of interleukin 8 (CXCL8) in human intestinal microvascular endothelial cells are mediated by CXCR2. J Biol Chem. 2003;278(10):8508-15.

26. Qian Y, Wang Y, Li DS, Zhu YX, Lu ZW, Ji QH, et al. The chemokine receptorCXCR2 plays a critical role in the invasion and metastases of oral squamous cell carcinoma in vitro and in vivo. J Oral Pathol Med. 2014;43(9):658-66.

27. Wang B, Hendricks DT, Wamunyokoli F, Parker MI. A growth-related oncogene/CXC chemokine receptor 2 autocrine loop contributes to cellular proliferation in esophageal cancer. Cancer Res. 2006;66(6):3071-7.

28. Halpern JL, Kilbarger A, Lynch CC. Mesenchymal stem cells promote mammary cancer cell migration in vitro via the CXCR2 receptor. Cancer Lett. 2011;308(1):91-9.

29. Murdoch C, Monk PN, Finn A. Cxc chemokine receptor expression on human endothelial cells. Cytokine. 1999;11(9):704-12.

30. Fuse N, Kuboki Y, Kuwata T, Nishina T, Kadowaki S, Shinozaki E, Machida N, Yuki S, Ooki A, Kajiura S, et al. Prognostic impact of HER2, EGFR, and c-MET status on overall survival of advanced gastric cancer patients. Gastric cancer. 2015. Epub ahead of print.

31. Kwon CH, Park HJ, Lee JR, Kim HK, Jeon TY, Jo HJ, et al. Serpin peptidase inhibitor clade A member 1 is a biomarker of poor prognosis in gastric cancer. Br J Cancer. 2014;111(10):1993-2002. 